Research Article

\title{
Evaluation of the Influencing Factors of Using Underground Space of Abandoned Coal Mines to Store Hydrogen Based on the Improved ANP Method
}

\author{
Wang Liu $(\mathbb{D})$ and Peng Pei $\mathbb{C}$ \\ College of Mining, Guizhou University, Guiyang 550025, China \\ Correspondence should be addressed to Peng Pei; ppei@gzu.edu.cn
}

Received 9 November 2021; Accepted 9 December 2021; Published 28 December 2021

Academic Editor: Zhengyang Song

Copyright (c) 2021 Wang Liu and Peng Pei. This is an open access article distributed under the Creative Commons Attribution License, which permits unrestricted use, distribution, and reproduction in any medium, provided the original work is properly cited.

\begin{abstract}
Storage is currently a major obstacle to the promotion of hydrogen energy. Hydrogen storage in abandoned coal mines can achieve the effective use of underground space while meeting the growing demand for energy storage facilities, which can bring economic and environmental benefits. However, research in this area has been limited to the conceptual discussion stage, without establishing a scientific evaluation method for the potential of modifying and utilizing abandoned coal mine space. In this study, based on the analytic network process (ANP), the Apriori algorithm is introduced to mine the association rules for various influencing factors. First, the Apriori algorithm is applied to mine association rules between indicators, eliminate unnecessary influence relationships, simplify the network structure model, and optimize the ANP weight calculation results; second, the solution method of judgment matrix is improved with triangular fuzzy numbers, and the index weight is solved by fuzzy nine marks instead of the method of nine scale, which is convenient for experts to give the fuzzy scale while better reflecting the opinions of experts. Finally, the ANP algorithm is applied to rank the weights of the obtained influencing factors, discuss the main factors with higher weights, and analyze the feasibility of converting candidate coal mines into hydrogen storage facilities using the derived evaluation method in the case study. The evaluation methods and conclusions presented in this study provide analytical tools and a decision basis for analyzing the feasibility of converting underground space of abandoned coal mines into hydrogen storage facilities and assessing the economic indicators.
\end{abstract}

\section{Introduction}

With the attention of controlling greenhouse gas emission and guarantee of stable supply of energy, the development of new technology utilizing clean energy sources has been expedited to mitigate energy shortage risk and restore the natural ecology [1-3]. Economical, large-scale energy storage technology plays a key role in enabling the utility industry to integrate more renewable energy sources into the grid [4]. Many countries have started to set targets for the utilization of hydrogen energy in industry, construction, electricity, and transportation sectors [5]. By using excess electricity generated by the window and solar power that cannot be accepted by the grid to electrolyze water into oxygen and hydrogen, the hydrogen production cost can be significantly reduced The concept of a hydrogen energy chain is also expected to significantly reduce $\mathrm{CO}_{2}$ emissions [6]. Hydrogen energy is regarded as the future direction of clean energy research and development due to its clean, efficient, zero-carbon, and sustainable utilization. However, the development of hydrogen energy still faces many challenges, of which storage is the most critical one [7]. Large-scale hydrogen storage may be achieved in two ways: (1) by integrating hydrogen storage into renewable energy system; (2) by using "electricity-to-gas" technology, where excess electricity from renewable energy sources is converted to hydrogen by water electrolysis and then temporarily stored for use during peak demand [8], to accommodate daily and seasonal variations in demand for electricity generation and hydrogen supply. As a renewable energy 
source, hydrogen can be used to solve this imbalance between supply and demand [9, 10].

At present, the most commonly used hydrogen storage methods include the following: high-pressure gaseous storage, low-temperature liquid storage, and storage alloys [11]. High-pressure gaseous hydrogen storage has the advantages of low cost and faster filling and discharging, but it consumes a large amount of energy in the compression process and needs to be equipped with high-strength, pressure-resistant, and corrosion-resistant containers, which are subject to risks such as leakage and bursting [10]. The biggest advantage of low-temperature liquid hydrogen storage is the relatively high density of hydrogen storage, but liquid hydrogen is easy to volatilize, and the storage and transportation process needs low-temperature supporting equipment, which is costly. Moreover, liquid hydrogen is prone to thermal stratification when stored in a large storage reservoir, which may lead to boiling of liquid hydrogen and bursting of tanks. The hydrogen storage alloy has a larger hydrogen storage density and better safety performance compared to the above two methods $[5,6]$, but the performance is unstable and the service life span is not ideal after repeated uses [12].

Compared with the above methods of hydrogen storage, the advantages of underground hydrogen storage are as follows [13]: (1) high storage safety and low possibility of sudden fire risks; (2) smaller land footprint and convenient connection with ground equipment; (3) low equipment cost, high applicability, and suitable for the geological conditions in many countries and regions [14]. At present, underground hydrogen storage in salt caverns have been built in both the United States and the United Kingdom, and the feasibility of underground hydrogen storage has also been discussed by scholars [13] by referring to the practical experience of natural gas storage. However, the research content on the use of underground space for hydrogen storage in coal mines is still rare.

Coal is the main energy source in many countries $[15,16]$, and after a long period of mining, many of the mineable reserves have tended to be depleted or have been depleted and closed, and these abandoned coal mines offer a large amount of underground space, such as shafts, tunnels, chambers, and other underground space resources $[17,18]$. The use of underground space of abandoned coal mines to store hydrogen provides a new idea for the transformation, development, and utilization of closed mines.

Underground hydrogen storage brings benefits in making full use of underground storage space, improving energy efficiency, and reducing the cost of gas storage. It plays an important role in peak shaving and a safe and stable fuel supply. However, the use of underground space for hydrogen storage needs to consider many safety and economic cost factors, whose influencing power should be properly weighted in the feasibility study and site selection stage [19]. In this study, we use the Apriori algorithm set combined with the analytic network process (ANP) method to screen various influencing factors and find out the key influencing factors to evaluate the feasibility of hydrogen storage in underground coal mine space $[20,21]$.
However, when the ANP method is used to investigate associations among factors, the intricacy calculation procedure is significantly raised and a larger calculation capacity is required. The Apriori algorithm is the first association rule mining algorithm and the most used one [21]. It divides and finds out associations by an iterative method of layer-bylayer search and is now commonly used in the creation of mathematical evaluation models. When combined with the ANP method, the associations of different elements can be first mined out by the Apriori algorithm, eliminating elements of relatively low influencing power, hence making the ANP calculation process more concise. This method has been used in evaluating safety risk factors in the power grid and transport sectors [22, 23].

The purpose of this study is to analyze and study the influencing factors of establishing hydrogen storage facilities in the underground space of abandoned coal mines using the Apriori algorithm in combination with the analytic network process (ANP) analysis method. In the feasibility study of converting abandoned coal mine space into a hydrogen storage facility, many factors and their associations need to be evaluated, including geological factors, gas storage conditions, and human technology factors. A traditional method such as the analytical hierarchy process cannot satisfy the requirement of accuracy. In this study, the ANP method improved by the Apriori algorithm is used to evaluate and rank the influencing factors of underground hydrogen storage in abandoned coal mines. The method presented in this study offers sufficient accuracy while being concise and can be used as a reliable approach in feasibility study and site selection of utilizing abandoned coal mines to the construction of underground hydrogen storage facility.

\section{Influencing Factors of Underground Hydrogen Storage in Abandoned Coal Mines}

Major factors affecting hydrogen storage in the underground space of abandoned coal mines can be generally classified into 3 categories, namely, geological factors, gas storage conditions, and human technology factors, as shown in Figure 1 .

\subsection{Geological Factors}

2.1.1. Development of Faults/Fractures. The main components of fractures surrounding rock are tectonic fractures, primary fractures, and secondary fractures [20]. Primary fractures are not affected by tunnel excavation. [25] Secondary fractures and tectonic fractures will further develop after being disturbed by tunnel excavation. The permeability of the rock body is more contributed by secondary and tectonic fractures [26]. Therefore, the degree of fracture development of the surrounding rock mass directly determines its permeability, which has an important influence on the safety of hydrogen storage facilities.

2.1.2. Rock Mass Stability. After the coal mine has been mined, the original rock stress state is altered. When the underground space is used as a hydrogen storage facility, the 


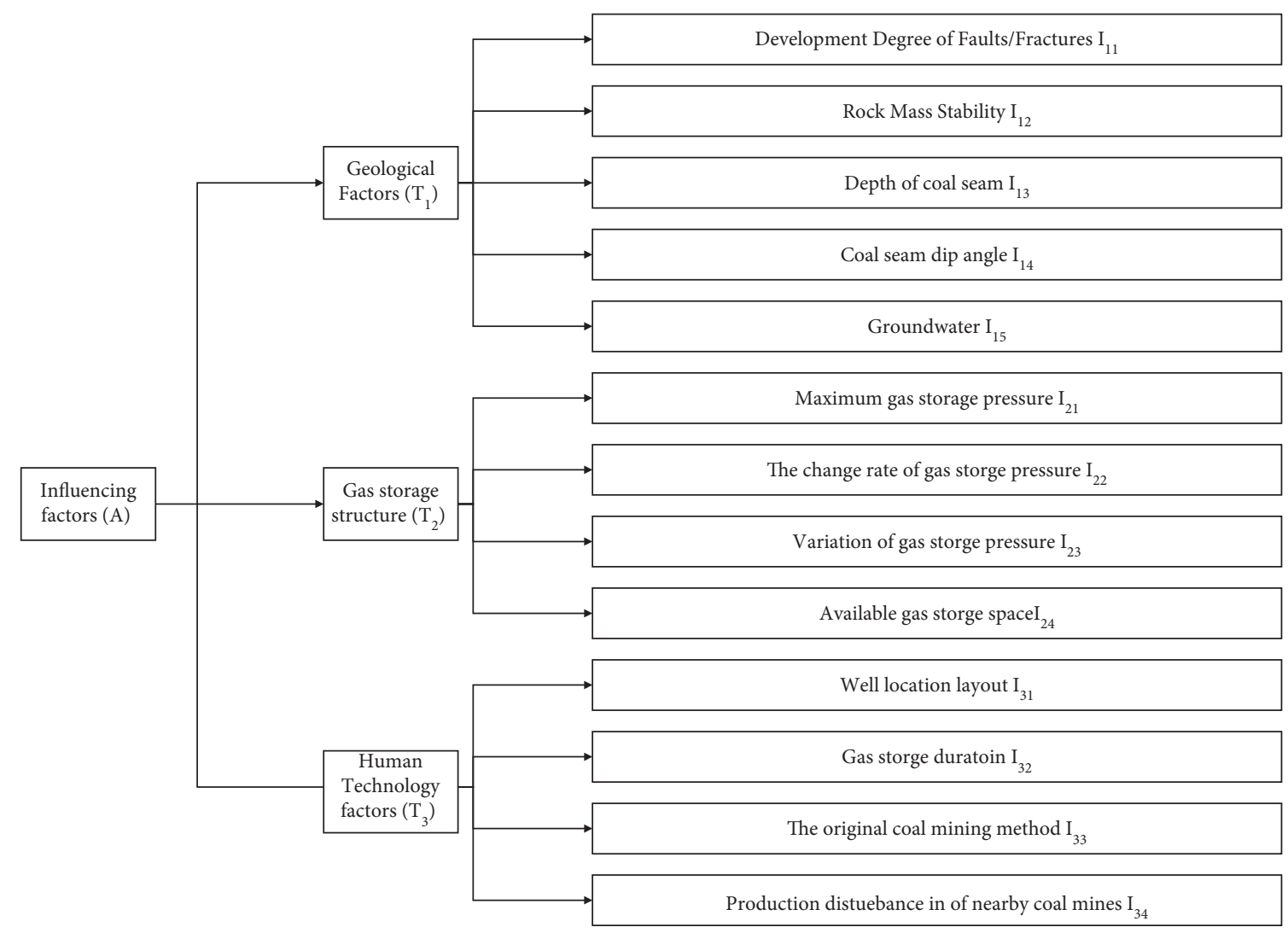

FIGURE 1: Influencing factors of underground hydrogen storage in abandoned coal mines.

stress state and stability of the rock mass are also changed by the periodic pressure variation due to gas injection and discharging [26], and this will induce an impact on the stability of hydrogen storage facilities.

2.1.3. Depth of Coal Seam. When the coal seam depth is shallow, the overlying rock layer on the working surface is easy to produce subsidence; the overlying rock stability in the mining area is poor; and the coal seam burial depth determines the size, direction, and distribution state of the original rock stress, and the maximum allowable gas storage pressure, which affects the gas storage conditions and the stress state of the surrounding rock.

2.1.4. Coal Seam Dip Angle. The coal seam dip angle affects the damage state of the roof of the goaf. When the coal seam dip angle is larger, the overburden stress distribution of the overlying rock in the goaf is more complicated and the fissures and fractures are more developed, so the stability is poor [16].

2.1.5. Groundwater. When groundwater enters the storage and conductive spaces within the rock, it changes the stress state and mechanical properties of the rock mass; the available space for gas storage is reduced, and the risk is increased.

\subsection{Gas Storage Conditions}

2.2.1. Maximum Gas Storage Pressure. Due to the complexity of the geological condition of coal mines, the effective stress of the rock mass will also change during the process of injecting and pumping hydrogen gas. When the gas storage pressure is greater than the in situ stress of the surrounding rock, the surrounding rock is in a tensile state, which affects the stability of the rock mass. In addition, due to a large number of mechanically weak surfaces in the rock mass in the extraction area, the weak surface might expand after the construction of the hydrogen storage reservoir, which has an important influence on the stability of the hydrogen storage reservoir.

2.2.2. The Rate of Change of Gas Storage Pressure. In the process of injecting and discharging hydrogen, the rock mass is subjected to alternating stress cycles, and the transformation rate of storage pressure affects the rate of change and frequency of effective stress in the surrounding rock, which forms an impact on the stability of the surrounding rock.

2.2.3. Variation of Gas Storage Pressure. Under the longterm alternating stress induced by the injection and discharging cycle, the mechanical properties of rock mass will be altered, and the magnitude of the stress change is one of the influencing factors. 
2.2.4. Available Gas Storage Space. The underground space of abandoned coal mines mainly includes shafts, roadways, chambers, and goafs. Not all spaces are available for the establishment of hydrogen storage, so the effective available space hydrogen storage affects the feasibility of the construction of hydrogen storage reservoirs.

\subsection{Human Technology Factors}

2.3.1. Well Location Layout. According to different injection and pumping requirements and gas storage conditions, well positions need to be arranged according to specific conditions. Different well placements will determine the gas flow in the storage space, which will affect the injection and pumping efficiency and economic cost.

2.3.2. Gas Storage Duration. The gas storage duration determines the frequency of stress changes in the surrounding rock and affects the stability of the surrounding rock.

\subsubsection{The Original Coal Mining Method. Different mining} methods lead to different exposed areas of the goaf roof. For example, in room and pillar mining, the exposed area of the goaf is relatively small and it is conducive to the stability of the hydrogen storage structure; while in the long-arm type of mining, the exposed area of the goaf is larger and it is not conducive to the stability of the hydrogen storage structure.

2.3.4. Stress Disturbance of Nearby Coal Mines. The stress disturbance generated by blasting vibrations in the excavation and production process of the nearby coal mines and the induced stresses generated by the mining may be transferred to the surrounding rocks of the hydrogen storage reservoir, which may superimpose with the stresses on the surrounding rocks of the reservoir itself and affect the stability of the gas storage structure.

2.3.5. Airtightness Treatment. In order to prevent leakage in the process of hydrogen storage, it is necessary to conduct airtightness treatment of the underground space. Therefore, it is an important factor in the evaluation of the feasibility of underground hydrogen storage in abandoned coal mines [27].

\section{Apriori Algorithm and Ascertainment of Algorithm}

Because of the uncertainty of the correlation of factors in the ANP method, the interrelationships between indicators, association rules, and elimination of unnecessary factors are mined by the Apriori algorithm on the basis of the internal influencing factors determined by experts [28], hence simplifying the network structure of ANP.

The influence relationship between the indicators is set as determined by the expert method as a data set $\{D\}$, each indicator group $T_{i}(i=1,2,3, \ldots, 5)$ is the affairs in $\{D\}$, and the indicators $i_{i j}(i, j=1,2, \ldots, 5)$ are the items of $T_{i}$, where $I$ is the set of all indicators composed of $\{D\}$, and any subset $\{X\}=K$ is the itemset of $\{D\}$. Then, the support degree of subset $\{X\}[16,20,21]$ is formulated as

$$
\operatorname{sub}(X)=\frac{\delta(X)}{D} \times 100 \%
$$

Here, $\delta(X)$ is the support of itemset $\{X\}$ in $\{D\}$. For $X$ that satisfies the specified minimum support, $\operatorname{Sup}(X)$ requirement is called the set of frequent $K$ items of $\{D\}$.

The confidence coefficient of association rule $X \longrightarrow Y$ refers to the probability that not only the itemset $X$ but also the itemset $Y$ is formulated as

$$
\operatorname{con}(X \Rightarrow Y)=\frac{\operatorname{sub}(X \cap Y)}{\operatorname{sub}(X)} \times 100 \% \text {. }
$$

The minimum support minsub $(X)$ and the minimum confidence coefficient $(X \longrightarrow Y)$ are used to express the minimum importance according to the fixed rules and the minimum confidence that must be met [29]. When the minimum support and minimum credibility requirements are met at the same time, the association degree is established; otherwise, the association rules are redundant, and the association relationship is eliminated.

The example of finding the association rule of indicator $I_{22}$ in $T_{2}$ is taken, the minsub $(X)=40 \%$ and coefficient $(X \longrightarrow Y)=70 \%$ are defined [50], to simplify the calculation process, and indicators that do not affect each other are selected based on expert experience, as shown in Table 1.

In order to simplify the calculation process, the relevant itemsets are calculated using Python. The calculation result is $\left\{I_{11}, I_{12}, I_{15}, I_{35}, I_{36}\right\}$.

Similarly, the set of association rules for each indicator is shown in Table 2.

\section{Evaluation of Influencing Factors}

4.1. Analytic Network Process (ANP) Method. Analytic network process (ANP) is a complex system evaluation and decision-making method [31]. ANP divides the system elements into two major parts. The first part becomes the control factor layer, which is independent from each other and controlled by the target element. The second part is the network layer, which consists of all the interrelated and mutually influencing elements governed by the control layer, and Figure 2 is a typical ANP structure.

4.2. F-ANP Theory and Calculation Results. The ANP method is used to establish a judgment matrix by comparing the magnitude of the interaction relationship between indicators by expert scoring two by two. In the decisionmaking process, the complexity and uncertainty of objects should be considered, and the judgment value obtained when constructing the ANP judgment matrix is not a fixed numerical point and may vary within a certain interval. Therefore, this study adopts the F-ANP method, which is a combination of fuzzy evaluation method and ANP method, to evaluate the influencing factors, and uses the triangular fuzzy function to deal with the uncertainty of the influence 
TABLE 1: Related $I_{22}$ information of 1 itemset.

\begin{tabular}{lc}
\hline Expert number & $I_{22}$-related indicators \\
\hline A1 & $I_{11} I_{12} I_{15} I_{24} I_{34} I_{35}$ \\
A2 & $I_{11} I_{12} I_{13} I_{15} I_{33} I_{34} I_{35}$ \\
A3 & $I_{11} I_{12} I_{15} I_{24} I_{31} I_{32} I_{35}$ \\
A4 & $I_{11} I_{12} I_{15} I_{35}$ \\
A5 & $I_{11} I_{12} I_{15} I_{33} I_{34} I_{35}$ \\
\hline
\end{tabular}

TABLE 2: Set of association rules among various indicators.

\begin{tabular}{lc}
\hline Index & Indicators with associated rules \\
\hline$I_{11}$ & $I_{12} I_{15} I_{21} I_{33}$ \\
$I_{12}$ & $I_{11} I_{14} I_{15} I_{21} I_{23} I_{33}$ \\
$I_{13}$ & $I_{11} I_{12} I_{14}$ \\
$I_{14}$ & $I_{11} I_{12} I_{13} I_{15} I_{33}$ \\
$I_{15}$ & $I_{11} I_{13} I_{33}$ \\
$I_{21}$ & $I_{11} I_{12} I_{35}$ \\
$I_{22}$ & $I_{11} I_{12} I_{15} I_{35}$ \\
$I_{23}$ & $I_{11} I_{12} I_{15} I_{35}$ \\
$I_{24}$ & $I_{11} I_{12} I_{13} I_{14} I_{15} I_{31} I_{33} I_{34} I_{35}$ \\
$I_{31}$ & $I_{12} I_{13} I_{14} I_{15} I_{33}$ \\
$I_{32}$ & $I_{11} I_{21} I_{24} I_{35}$ \\
$I_{33}$ & $I_{11} I_{12} I_{13} I_{14} I_{15} I_{34}$ \\
$I_{34}$ & $I_{31} I_{33} I_{36}$ \\
$I_{35}$ & $I_{11} I_{21} I_{24} I_{34}$ \\
\hline
\end{tabular}

relationship between indicators. The importance of indicators is not determined by a definite scale, but it is reflected by a triangular fuzzy function [32].

The triangular fuzzy number $\tilde{x}$, which means "approximately being $x$," and the corresponding triangular fuzzy function are formulated as follows:

$$
\tilde{X}= \begin{cases}(1,2,3), & X=1, \\ (X-1, X, X+1), & 2 \leq X \leq 8 \\ (8,9,9)^{\prime 12}, & X=9\end{cases}
$$

In F-ANP, the marks are judged by the method of nine marks and redefined as fuzzy nine marks to judge the comparison marks. The method of fuzzy nine marks improved by the triangular fuzzy function can not only more accurately reflect expert opinions but also the expression form that is closer to the typical, and there are certain advantages in algorithm application and computation. The comparison marks judged by fuzzy nine marks are shown in Table 3.

The triangular fuzzy number $\tilde{x}$ defines the trigonometric function, as shown in Table 3.

When constructing the judgment matrix, if the expert can clearly judge whether the importance degree is greater or less than $x$ in addition to the index importance mark being $x$, then the importance information of the index can be more clarified. In order to further clarify the expert opinion [33], this study will define fuzzy numbers that may be larger than $x$ and smaller than $x+1$, which are defined as $\tilde{x}^{+}$; fuzzy numbers that may be smaller than $x$ and larger than $\mathrm{x}-1$ are defined as $\tilde{x}$.
If the probability of any two fuzzy numbers $b_{i}$ and $b_{j}$ set is $\mathrm{V}\left(\widetilde{\mathrm{b}}_{i} \geq \widetilde{\mathrm{b}}_{j}\right)$, then the formulas are as follows:

$$
v\left(\widetilde{b}_{i} \geq \widetilde{b}_{i}\right)\left\{\begin{array}{l}
1, b_{i}^{m}=b_{j}^{m}, \\
\frac{b_{i}^{n}-b_{j}^{1},}{\left(b_{i}^{n}-b_{i}^{m}\right)+\left(b_{j}^{m}-b_{j}^{1}\right),} \\
0, b_{i}^{n} \leq b_{j}^{1} .
\end{array}\right.
$$

In formula (4), $b^{n}$ and $b^{1}$ are the upper limit and lower limit of $b ; b^{m}$ is the median value of $b$ and $b^{1} \leq b^{m} \leq b^{n}$.

If the ambiguity function is defined, then the potential degree of $b_{i} \geq b_{j}$ is $\mathrm{P}\left(b_{i} \geq b_{j}\right)$ in the formula (5):

$$
P\left(\widetilde{b}_{i} \geq \tilde{b}_{j}\right)=\frac{\left(V\left(\widetilde{b}_{i} \geq \tilde{b}_{j}\right)+1-V\left(\widetilde{b}_{j} \geq \widetilde{b}_{i}\right)\right.}{2} .
$$

The element $r_{i j}=p\left(b_{i} \geq b_{j}\right)$, where $i, j=1,2,3, \ldots, n$. Complementary matrix $R=\left(r_{i j}\right)_{n \times n}, r_{i j}=1-r_{i j}$, and $r_{i j}=0.5$; then, the weight $(\mathrm{Wi})$ of the $i$-th index is shown in

$$
W_{i}=\frac{1}{n(n-1)}\left(\sum_{j=1}^{n} r_{i j}+\frac{n}{2}-1\right) .
$$

When applying the ANP method to construct the judgment matrix $[34,35]$, each indicator is used as the subcriteria, and the two comparisons are made between the remaining indicators under the already established association rules, and the magnitude of influence is expressed by the triangular fuzzy number. Here, taking the $I_{12}$ index as an example, the remaining elements in $T_{2}$ are compared in pairs, respectively, to determine the degree of influence on $I_{12}$, and a judgment matrix is constructed. The consistency is checked, and the judgment matrix is solved according to the formula, as shown in Table 4.

For the judgment matrix that satisfies the consistency check, the ranking vector value $\omega_{i}$ in the matrix is used as the fuzzy ranking vector of each index group in the index group $T_{1}$ to construct the fuzzy weight matrix $A=\{\alpha \mathrm{ij}\}$, and the unweighted fuzzy supermatrix $W$ is constructed from the index fuzzy ranking vector $=\left\{\omega_{i j}\right\}$. When there is no correlation between the indicators, the influence degree is set to be 0 .

$\mathrm{A}$ and $\mathrm{W}$ are multiplied to solve the fuzzy weighted hypermatrix $\mathrm{W}^{\prime}$, that is, $\mathrm{W}^{\prime}=\alpha_{i} \times \omega_{i j}$. Each element of the matrix in $\mathrm{W}^{\prime}$ is the fuzzy weight value of the grid index in the index system. In order to reflect the interrelationship between the elements, the limit relative fuzzy weight vector is calculated for the hypermatrix $\mathrm{W}^{\prime}$ with

$$
W^{\prime \prime}=\lim _{n \longrightarrow \infty}\left(W^{\prime}\right)^{n}
$$

Finally, a unique and convergent matrix $\mathrm{W}^{\prime}$ is obtained, and the column vector of $\mathrm{W}^{\prime}$ is obtained, which is the index limit fuzzy weight of $I_{i j}$. 


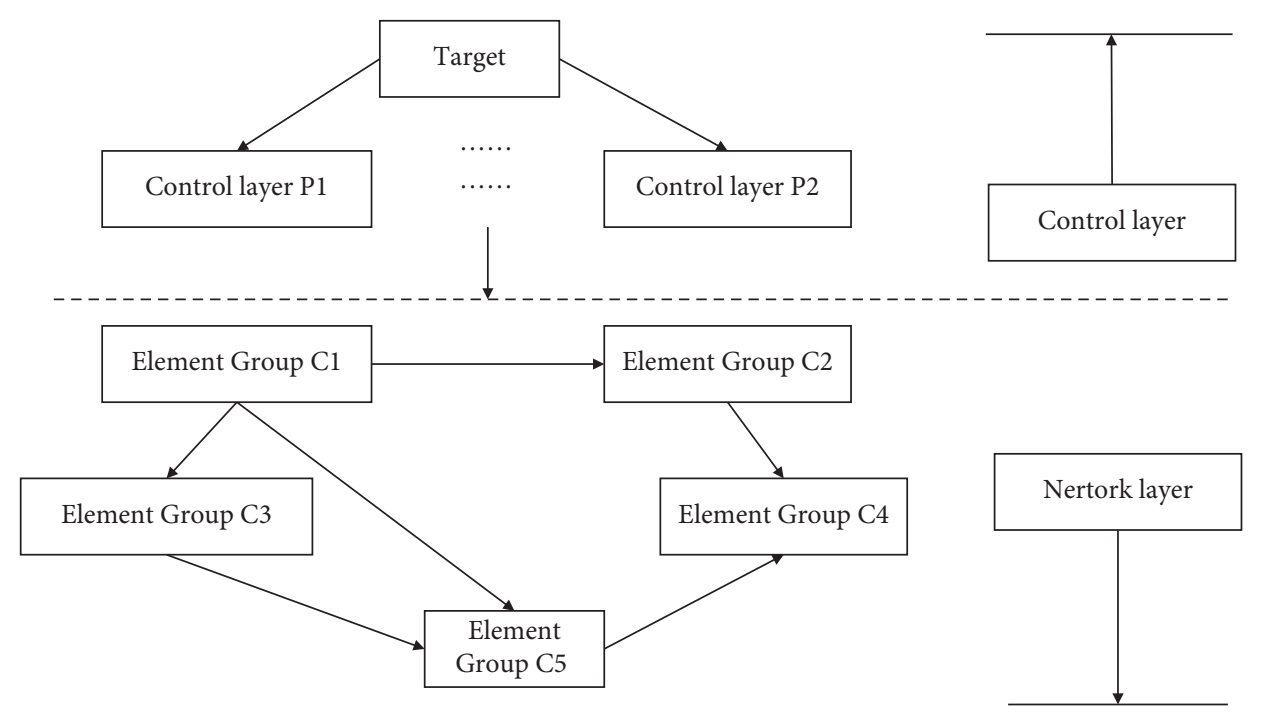

FIgURE 2: ANP structure.

TABLE 3: Fuzzy nine marks.

\begin{tabular}{lc}
\hline Compared importance of $x_{i}$ and $x_{j}$ & $\widetilde{x}_{i j}$ \\
\hline Equally important & $\widetilde{1}$ \\
More important & $\widetilde{3}$ \\
Obviously important & $\widetilde{5}$ \\
Strongly important & $\widetilde{7}$ \\
Extremely important & $\widetilde{9}$ \\
In the middle & $\widetilde{2}, \widetilde{4}, \widetilde{6}, \widetilde{8}$ \\
\hline
\end{tabular}

TABle 4: Pairwise judgment matrix of $I_{12}$ in $T_{1}$.

\begin{tabular}{lcccc}
\hline$I_{12}$ & $I_{11}$ & $I_{14}$ & $I_{15}$ & $\omega_{i}$ \\
\hline$I_{11}$ & 1 & $\widetilde{2}$ & $\widetilde{2}$ & 0.39734 \\
$I_{14}$ & $\widetilde{2}^{-1}$ & 1 & $\widetilde{3}$ & 0.38564 \\
$I_{15}$ & $\widetilde{2}^{-1}$ & $\widetilde{3}^{-1}$ & 1 & 0.21702 \\
\hline
\end{tabular}

Finally, the final obtained weights of each indicator are ranked in Table 5 .

4.3. Case Analysis. The influencing factors selected by the above methods can be used to sort and select the candidate sites of abandoned coal mines for constructing hydrogen storage facilities. This section will introduce a case study to illustrate this process. This case selects 10 mines in Guizhou Province, western China, clarifies the degree of influencing factors, as shown in Table 6, and then screens the 10 mines according to the ranking of influencing factors. The candidate sites are screened following the order of influencing factors mentioned in Table 5 in Section $4.2 I_{24}, I_{35}, I_{12}, I_{21}, I_{11}$, and $I_{32}$. First, the most important influencing factor $I_{24}$ is compared. Then, the corresponding factors of the passed sites are compared. Finally, the most appropriate site is screened out. In the case study, CM3 is the most appropriate. By this screening method, the site selection process becomes more reasonable and intuitive.

\section{Discussion}

The above calculation results show that $I_{24}, I_{35}$, and $I_{12}$ are important factors affecting hydrogen storage, and $I_{11}, I_{21}$, and $I_{32}$ are more important factors affecting hydrogen storage. The specific discussion and analysis are as follows.

5.1. The Most Important Influencing Indicators. Indicator $I_{24}$ is the available gas storage space. It occupies the highest weight in the index. The main reason is to maximize underground benefits, and a sufficient gas storage space is necessary. If the hydrogen storage space is insufficient, the basic functions of the hydrogen storage facilities cannot be guaranteed, and the economic benefits are not good enough.

Indicator $I_{35}$ is the airtightness treatment. The reason is that if the airtightness treatment is insufficient, hydrogen will escape and cannot be stored in large quantities. As a flammable and explosive gas, it will also increase the risk factor of hydrogen storage facilities. Hydrogen escaped into fracture has the potential to rejuvenate fractures and fissures, 
TABLE 5: Fuzzy weight ranking of each indicator.

\begin{tabular}{lcc}
\hline Index & Index weight & Weight ranking \\
\hline$I_{11}$ & 0.08708 & 5 \\
$I_{12}$ & 0.09239 & 3 \\
$I_{13}$ & 0.07006 & 7 \\
$I_{14}$ & 0.06553 & 8 \\
$I_{15}$ & 0.03225 & 13 \\
$I_{21}$ & 0.08837 & 4 \\
$I_{22}$ & 0.05669 & 9 \\
$I_{23}$ & 0.05662 & 10 \\
$I_{24}$ & 0.16305 & 1 \\
$I_{31}$ & 0.01642 & 14 \\
$I_{32}$ & 0.08663 & 6 \\
$I_{33}$ & 0.03553 & 12 \\
$I_{34}$ & 0.04863 & 11 \\
$I_{35}$ & 0.09598 & 2 \\
\hline
\end{tabular}

TABLE 6: Information study on the degree of influencing factors of the coal mine.

\begin{tabular}{|c|c|c|c|c|c|c|}
\hline & $\begin{array}{l}\text { Development degree } \\
\text { of fault fissure }\end{array}$ & $\begin{array}{l}\text { Rock mass } \\
\text { stability }\end{array}$ & $\begin{array}{l}\text { Maximum gas } \\
\text { storage pressure }\end{array}$ & $\begin{array}{l}\text { Available gas } \\
\text { storage space }\end{array}$ & $\begin{array}{c}\text { Gas storage } \\
\text { duration }\end{array}$ & $\begin{array}{l}\text { Airtightness } \\
\text { treatment }\end{array}$ \\
\hline CM1 & High & Poor & Higher & Large & Long & Good \\
\hline CM2 & Lower & Moderate & Higher & Small & Short & Good \\
\hline CM3 & Low & Better & High & Larger & Moderate & Good \\
\hline CM4 & Higher & Good & Low & Medium & Longer & Better \\
\hline CM5 & Higher & Good & Lower & Medium & Shorter & Weak \\
\hline CM6 & High & Difference & Medium & Smaller & Long & Good \\
\hline CM7 & High & Better & Medium & Small & Long & Better \\
\hline CM8 & Medium & Difference & Low & Bigger & Shorter & Poor \\
\hline CM9 & Higher & Better & Higher & Bigger & Longer & Poor \\
\hline CM10 & Lower & Good & High & Small & Longer & Good \\
\hline
\end{tabular}

reducing the effective stress of rock mass and weakening its mechanical strength.

Indicator $I_{12}$ is the rock mass stability. The reason is that the stress state and stability of the rock mass change under the influence of the periodic stress of gas injection and pumping. In order to ensure the safety of hydrogen storage facilities, the cost of reconstruction will be increased, which will affect the efficiency of hydrogen storage and have an important impact on the feasibility of hydrogen storage.

5.2. The Second Most Importance of Influencing Indicators. Indicator $I_{21}$ is the maximum gas storage pressure. The reason is that when the maximum gas storage pressure is greater than the stress of the surrounding rock, the surrounding rock will be in a tensile state. Since the tensile strength of rock is typically low, the stability of the rock mass will be severely impacted, thereby affecting the safety of the hydrogen storage facility. Moreover, if hydrogen cannot be stored at sufficient pressure, the cost of pumping to discharge gas will increase, which will affect the efficiency of hydrogen storage.

Indicator $I_{11}$ is the fault fissure development degree. The reason is that the development degree of the surrounding rock fractures and fissure directly determines the permeability and mechanical stability of the surrounding rock, which increases the difficulty and cost of antiseepage treatment, and has a significant impact on hydrogen storage facilities.

Indicator $I_{32}$ is the gas storage duration. The reason is that the gas storage duration determines the frequency of injection and discharging of hydrogen storage facilities and affects the frequency of the surrounding rock stress at the same time, which has an impact on the stability of the surrounding rock. It increases the cost of safety input and affects the efficiency of hydrogen storage.

The method provides an approach to evaluate the influencing factors and rank site. However, factors considered here might not be universal and should be supplemented in practice cases.

\section{Conclusions}

Evaluating the influencing factors of hydrogen storage in the underground space of abandoned coal mines has an important role in the reuse of abandoned coal mines and in providing an option for hydrogen storage on a large scale. This study establishes a system for evaluating the major influencing factors for assessing hydrogen storage in underground space reconstruction and the structural characteristics of underground abandoned space and uses network analysis to calculate their values. The conclusions are as follows: 
(1) When using the underground space for hydrogen storage in abandoned coal mines, there are many factors that need to be considered, among which, the available gas storage space, airtightness treatment, and rock mass stability are the most important, followed by the maximum storage pressure, the degree of fault fracture development, and the storage duration.

(2) The study analyzes the influencing factors of hydrogen storage in underground coal mines and establishes an evaluation index system. The feasibility of hydrogen storage by using abandoned coal mines and its potential for hydrogen storage are evaluated. The research result provides a feasible evaluation system for hydrogen storage potential in the underground space of abandoned mines.

(3) The study introduces the reasons for the importance of the influencing factors of underground hydrogen storage and analyzes the difference in the weight of the influencing factors. It can be used as a research tool for future evaluation of the modification potential of underground space.

(4) The research methods and conclusions in this study can further promote the renovation and utilization of underground space in abandoned mines.

\section{Nomenclature}

Symbols

A: Target layer

$T$ : Standard layer

$C$ : Indicator layer

I: $\quad$ Factor level

$x$ : Fuzzy scale

$\alpha_{i j}$ : Judgment matrix element.

Greek letters

$\omega_{i j}$ : Weight vector.

Abbreviations

AHP: Analytic hierarchy process

ANP: Analytic network process.

\section{Data Availability}

All data, models, and codes generated or used during the study are included within the article.

\section{Conflicts of Interest}

The authors declare that they have no conflicts of interest.

\section{Acknowledgments}

This work was supported by the National Natural Science Foundation of China (grant number: 52066005) and the Natural Science Foundation of Guizhou Province (grant number: [2020]2Y025).

\section{References}

[1] X. Feiran, J. Zhao, and S. Jiang, "Building thermal comfort research based on energy-saving concept," Advances in $\mathrm{Ma}$ terials Science and Engineering, vol. 2021, Article ID 7132437, 11 pages, 2021.

[2] L. Ruifei, Z. Jin, and K. Peter, "Consumption- and incomebased sectoral emissions of polycyclic aromatic hydrocarbons in China from 2002 to 2017," Environmental Science \& Technology, vol. 55, no. 6, 2021.

[3] W. Yang, Z. Wang, H. Pei, J. Zhang, and P. Krebs, "Impact of green infrastructure on the mitigation of road-deposited sediment induced stormwater pollution," The Science of the Total Environment, vol. 770, 2021.

[4] C. L. Chu, C. F. Chang, J. R. Chen, and Y. K. Fuh, "Hydrogen storage in iron/carbon nanopowder composite materials: effect of varying spiked iron content on hydrogen adsorption," Journal of Nanomaterials, vol. 2013, Article ID 742075, 7 pages, 2013.

[5] P. Pei, S. F. Korom, K. Ling, J. He, and A Gil, "Thermodynamic impact of aquifer permeability on the performance of a compressed air energy storage plant," Energy Conversion and Management, vol. 97, pp. 340-350, 2015.

[6] B. P. U. Roy and N. Rengarajan, "Feasibility study of an energy storage system for distributed generation system in islanding mode," ASME. J. Energy Resour. Technol. January, vol. 139, no. 1, 2017.

[7] H. Qing and S. Yi, "Development status of wind-hydrogen coupled energy storage system technology," Thermal Power Generation, vol. 50, pp. 9-17, 2021.

[8] Y. Liang, J. Yaodong, W. Kai, Z. Yixin, H. Xianjie, and $\mathrm{X}$. Chao, "Scientific thinking on the precise development and utilization of closed/abandoned mine resources in my country," Journal of China Coal Society, vol. 43, no. 1, pp. 14-20, 2018.

[9] C. R. Matos, J. F. Carneiro, and P. P. Silva, "Overview of largescale underground energy storage technologies for integration of renewable energies and criteria for reservoir identification," Journal of Energy Storage, vol. 21, pp. 241-258, 2019.

[10] W. Wei, "Research on the joint system of intermittent renewable energy power generation-hydrogen production and energy storage," master Thesis, Huazhong University of Science and Technology, Hangzhou, China, 2016.

[11] Y. Hongmei, S. Zhigang, and H. Ming, "Research progress and development suggestions for hydrogen production by electrolysis of water," Engineering Science in China, vol. 23, no. 2, pp. 1-7, 2021.

[12] Y. Zheng, Y. Tan, C. Zhou et al., "A review on effect of hydrogen on rubber seals used in the high-pressure hydrogen infrastructure," International Journal of Hydrogen Energy, vol. 45 , no. 43, p. 23721, 2020.

[13] H. I. A. Ramadan, S. Haitham, A. S. Mohamed, and H. Daniel, "Hydrogen storage technologies for stationary and mobile applications: review, analysis and perspectives," Renewable and Sustainable Energy Reviews, vol. 149, 2021.

[14] T. Radosław, U. M. Barbara, and T. Piotr, "Storage of hydrogen, natural gas, and carbon dioxide - geological and legal conditions," International Journal of Hydrogen Energy, vol. 46, no. $38,2021$.

[15] B. Mingxing, S. Kaoping, X. Baocheng et al., "The feasibility, limitations and development prospects of underground hydrogen storage," Geological Review, vol. 60, no. 4, pp. 748-754, 2014. 
[16] J. Qianhui, "Discussion on the feasibility of underground infusion technology of abandoned coal mine waste_ Ji Qianhui," Coal Geology and Prospecting, vol. 42, no. 4, pp. 69-71+76, 2014.

[17] D. Wu, "Multi-physics coupling theory research on compressed air energy storage in abandoned coal mine underground space," Master Thesis, China University of Mining and Technology, Xuzhou,China, 2020.

[18] S. Dacheng, P. Peng, and Z. Yujun, "Techno-economic feasibility analysis of pumped storage hydroelectricity in abandoned underground coal mines," Journal of Energy Resources Technology, vol. 142, no. 12, 2020.

[19] W. Zhide, Z. Dewen, L. Dongxu, and D. Xuejie, "Feasibility study and suggestions on the construction of underground gas storage by using abandoned mines in my country," Coal Economic Research, vol. 39, no. 5, pp. 15-19, 2019.

[20] S. Qiang and J. Yuanyuan, "Hidden danger assessment for distribution network based on relativeentropy ANP and anti - entropy weight method," Journal of Shaoyang University, vol. 18, no. 4, pp. 14-23, 2021.

[21] X. zhu, L. Yao, and L. Ye, "Evaluationg of competitiveness of highway hub Cities Carrierbassed in ANP and Extension cloud model," Highways, vol. 66, no. 10, pp. 235-242 2021.

[22] L. Shuran and T. Qi, "Comprehensive evaluation of operation safety of large pumping station based on DEMATEL-ANPGRA," Journal of Safety and Environment, pp. 1-8, 2021.

[23] A. Sciacovelli, D. Smith, M. E. Navarro et al., "Performance analysis and detailed experimental results of the first liquid air energy storage plant in the world," Journal of Energy Resources Technology, vol. 140, no. 2, 2018.

[24] Z. Song, Y. Wang, H. Konietzky, and X. Cai, "Mechanical behavior of marble exposed to freeze-thaw-fatigue loading," International Journal of Rock Mechanics and Mining Sciences, vol. 138, 2021.

[25] Z. Zhonghao, Research on the Cause of Accident Based on AHP-Apriori Algorithm, Master Thesis, Jilin University, Jilin,China, 2015.

[26] A. L. Duigou and A. G. Bader, J. C. Lanoix and L. Nadau, "Relevance and costs of large scale underground hydrogen storage in France," International Journal of Hydrogen Energy, vol. 42, no. 36, 2017.

[27] W. Xuelei and M. Guomin, "Methods and development of hydrogen storage," Journal of Science \& Technology Economics, vol. 26, no. 20, p. 137, 2018.

[28] C. Lei, "Analysis of grouting reinforcement mechanism and engineering application of fault fissure zone," Energy and Conservation, vol. 180, no. 9, pp. 163-165, 2020.

[29] T. Akbar, H. G. Amir, and S. Asadollah, "A survey of evolutionary computation for association rule mining," Information Sciences, vol. 524, pp. 318-352, 2020.

[30] Z. Jincai, L. Tianquan, and Z. Yuzhuo, "Research on permeability characteristics of fractured rock mass," Journal of China Coal Society, vol. 22, no. 5, pp. 35-39, 1997.

[31] L. Weifeng and H. Xia, "A triangular fuzzy number multiattribute decision-making method with preference for alternatives," Fuzzy Systems and Mathematics, vol. 27, no. 2, pp. 155-161, 2013.

[32] Z. Lan, Y. Shuhong, and J. Hongjin, "Research on comprehensive evaluation of microgrid planning based on improved ANP_Zhulan," Acta Solar Energy, vol. 41, no. 3, pp. 140-148, 2020.

[33] S. Dacheng and P. Peng, "Analysis of influencing factors of modification potential of abandoned coal mine into pumped storage power station," Journal of Energy Resources Technology, vol. 143, no. 11, 2021.

[34] D. Zhaoyang, L. Yanling, and L. Hailong, "Effectiveness evaluation of combat ring nodes in the equipment network system based on ANP," Firepower and Command Control, vol. 5, no. 1, pp. 128-133+138, 2020.

[35] C. Roberto, C. Júlio, C. George, A. Paulo, R. Paulo, and A. José, "Methodology for sub-commercial calculation of the potential energy storage capacity of hydrogen, natural gas, and compressed air in salt caves," Journal of Energy Resources Technology, vol. 142, no. 4, 2020. 